\title{
Isolation, identification and optimization of laccase from Alternaria alternata
}

\author{
Asha T. Thakkar, Shreyas A. Bhatt \\ Department of Life Sciences, Hemchandracharya North Gujarat University, Patan 384 265, India.
}

\begin{tabular}{l}
\hline ARTICLE INFO \\
\hline Article history: \\
Received on: November 21, 2019 \\
Accepted on: January 17, 2020 \\
Available online: May 26, 2020 \\
\hline
\end{tabular}

\section{Key words:}

Lignocellulose, guaiacol, laccase, fungi, optimization

\begin{abstract}
Laccase, lignin peroxidase, and manganese peroxidase have a synergistic effect on a wide range of recalcitrant compounds. Among them, laccase is polyphenol oxidase widely available in fungi, plant species, and insects. Laccase has an important role in effluent decoloration, detoxification of pulp bleaching, and bioremediation process. Screening was carried out to find new fungal isolate for the presence of laccase activity with guaiacol as indicator compound. Sixteen fungal isolates were obtained from biodeteriorated agro waste and the wood samples were collected from North Gujarat region of India. Among these isolates, one of the fungal isolates was observed with good laccase activity and identified as Alternaria alternata. Laccase activity was determined using 2,2'-azinobis-(3-ethylbenzethiazoline-6-sulfonate) as substrate. Various production parameters such as $\mathrm{pH}$, temperature, various carbon sources, nitrogen source, inducers, and cations were used to select the optimum condition for further increase in the production of this enzyme. Maximum laccase activity was obtained with glucose and sucrose as carbon source, $0.2 \%$ ammonium sulfate as nitrogen source, and $0.06 \% \mathrm{Cu}^{+2}$ with $1.5 \mathrm{mM}$ veratryl alcohol as inducer under optimized condition.
\end{abstract}

\section{INTRODUCTION}

The lifestyle of human can be improved by new innovations, research, and scientific achievements but ruins the environment differently. Different types of toxic chemicals which present in the environment are one of the major reasons for water toxicity which leads to adverse reaction on livelihood as well as on nature. Microbes have a massive capacity to reduce the toxicity of several types of chemicals with the help of enzymes. Laccase has diverse substrate flexibility which makes it highly interesting for diverse application, such as industrial development, pulp and textile dye bleaching, and bioremediation and detoxification of industrial effluents, and avoids environmental deteriorations [1-3]. Laccase has the most important impact on the reduction of diverse variety of pollutants, which is the thrust area to reduce environment pollution [2].

Laccases (benzenediol: dioxygen oxidoreductases, EC 1.10.3.2) are enzymes belonging to the class of blue oxidase-containing copper at catalytic site, composed of glycoproteins that oxidize a wide variety

\footnotetext{
*Corresponding Author

Shreyas A. Bhatt, Department of Life Sciences, Hemchandracharya North Gujarat University, Patan 384 265, India.

E-mail:sabhatt9@gmail.com
}

of phenolic compounds and aromatic amines and release free hydroxyl radicals for degradation of complexed compounds [2-5].

Lignin is the most recalcitrant components in lignocellulosic biomass that entraps the cellulose and hemicellulose. Pretreatment of lignocellulosic biomass is required to release fermentable sugar which can be accomplished by laccase [6]. Laccase plays a vital role in plant pathogenesis, pigment production, and lignocellulose degradation from agro waste [2]. The laccases from wood-rotting basidiomycetes are of significant interest as they have a potential to utilize different types of compounds such as aromatic compounds, phenols, and building blocks of lignin as carbon sources. Laccase also has a capacity to degrade lignin-mimicking compounds such as reactive, polymeric, and heterocyclic dyes [6].

Chemical pretreatment processes to release the fermentable sugars are expensive, difficult to operate, and environmentally unfriendly, and side-products after lignin breakdown during chemical delignification can lead to inhibitory effects on fermentation process. Depolymerization of lignin with the aid of laccases is a biological method which is far safer and economic alternatives to obtain fermentable sugars from lignocellulosic biomass [6]. 
The objective of the present study was to isolate and characterize laccase-producing fungi from agro waste and decayed wood material with the help of indicators to identify new laccase-producing fungal sources. Few studies were available for laccase in brown-rot fungi excluding reports on LiPs and MnPs activities [7]. Accordingly, as a part of this study, we have screened brown-rot fungal species to identify potent laccase producer and optimized enzyme production conditions for Ascomycetes species Alternaria alternata NS-6.

\section{MATERIALS AND METHODS}

\subsection{Materials}

Guaiacol and potato dextrose agar (PDA) were obtained from HiMedia. 2,2'-azinobis-(3-ethylbenzethiazoline-6-sulfonate) (ABTS), syringaldazine, and veratryl alcohol (3,4 dimethoxybenzyl alcohol) were acquired from Sigma-Aldrich (Germany). Analytical grade chemicals and reagents were used for the study.

\subsection{Screening and Isolation of Fungi}

A total of 5 decomposed wood samples and 25 agro waste samples were collected in sterile plastic bags from different areas of North Gujarat. To isolate lignolytic fungi, the samples were inoculated in potato dextrose broth with the use of lignin $(5 \% \mathrm{w} / \mathrm{v})$ as major carbon source along with glucose $(1 \% \mathrm{w} / \mathrm{v})$ for 5-7 days for the primary screening. The secondary screening was performed with the use of guaiacol $(0.02 \% \mathrm{v} / \mathrm{v})$ in PDA plate $[1,3,8]$. This plate assay helps to screen laccase-producing fungi by brown halos formation around colony. The medium was inoculated with fungal species and incubated for 7-10 days for the growth of fungi. A positive culture was subcultured when it shows positive result. One of the species, NS-6 isolate, which recorded with high production of brown color around colony, was selected for identification. Sequencing of the fungal isolate was carried out by Chromous Biotech (Bengaluru, India) using primers Internal Transcribed Spacer (ITS) 1 (5' TCCGTRSGNGAACYTGHGG $3^{\prime}$ ) and ITS 4 (5' TCCTCCGCTTATTKATDTGC 3') $[9,10]$ on an Applied Biosystem (ABI) 3500 XL Analyzer (USA), and the alignment of sequence was carried out using the Molecular Evolutionary Genetic Analysis version 6 (MEGA6) system software [11].

\subsection{Extracellular Laccase Activity}

Laccase activity was analyzed spectrophotometrically with a mixture containing $50 \mathrm{mM}$ ABTS in $100 \mathrm{mM} \mathrm{Na}$-Acetate buffer ( $\mathrm{pH}$ 5.0) with absorption maxima of $420 \mathrm{~nm}$ and an extinction coefficient of $36,000 \mathrm{M}^{-1} \mathrm{~cm}^{-1}$ [12-14]. The nonphenolic dye ABTS is converted to more stable state of cations which are responsible for intense green color $[15,16]$. Intense green color was observed due to more stable state of ABTS which was formed during reaction. Here, the laccase activity was determined with the use of suitable control and expressed as $\mathrm{U} / \mathrm{ml}$. About $1 \mu \mathrm{mol}$ of ABTS substrate oxidized per minutes is considered as one unit activity of laccase. The Bradford method was used to check the protein content of the sample [17].

\subsection{Cultivation of Fungal Isolate}

The quantitative determination of laccase activity was done by cultivation of fungal species in modified Kirk's medium, composed of (g/l): $\mathrm{MgSO}_{4} .7 \mathrm{H}_{2} \mathrm{O}(0.05), \mathrm{CaCl}_{2}(0.01), \mathrm{KH}_{2} \mathrm{PO}_{4}(0.20)$, glucose (10), thiamine (0.1), 2.2-dimethylsuccinic acid (2.90), ammonium tartrate (0.22), veratryl alcohol $(1.5 \mathrm{mM})$, and tween $80(0.10 \%$ $\mathrm{v} / \mathrm{v})$. Trace metal salt elements were also used with concentration (mg/l): $\mathrm{Fe}_{2}\left(\mathrm{SO}_{4}\right)_{3}(50), \mathrm{CuSO}_{4} .7 \mathrm{H}_{2} \mathrm{O}(80), \mathrm{H}_{2} \mathrm{MoO}_{4}(50), \mathrm{MnSO} 4$ (33), and $\mathrm{ZnSO}_{4} .7 \mathrm{H}_{2} \mathrm{O}(43)[18,19]$. Six agar plugs (cylindrical) were added from edge of mycelium growth in malt extract plate, and incubation was done at $30^{\circ} \mathrm{C}$ for 9 days. The samples were centrifuged at 9,500 $\mathrm{xg}$ for 10 minutes at $4^{\circ} \mathrm{C}$ temperature, and the crude laccase activity was analyzed from supernatants $[20,21]$.

\subsection{Optimization of Process Parameters}

The impact of various nitrogen and carbon compounds, such as nutrient sources, factors viz., $\mathrm{pH}$ and temperature, cations and inducers which were checked for optimum condition on laccase production by the fungus, was studied in a growth medium [17]. The laccase from the isolate NS-6 was investigated for its $\mathrm{pH}$ optima over a $\mathrm{pH}$ range of 2.5-6.5. The fungal isolate was incubated under optimum $\mathrm{pH}$ with the range of temperature from $25^{\circ} \mathrm{C}$ to $50^{\circ} \mathrm{C}$ for laccase production [15]. Nutrient source in form of carbon and nitrogen compounds was utilized for optimum growth of fungal isolates. Carbon sources such as glucose, maltose, mannitol, sucrose and lactose were used $(10 \% \mathrm{w} / \mathrm{v})$. Inorganic nitrogen sources, such as sodium nitrate and ammonium nitrate ammonium sulfate, and organic sources, such as peptone and yeast extracts were used at a final concentration of $0.2 \%$.

Different cations were used to find the most suitable cations for laccase production $[15,22]$. To check alternative source for veratryl alcohol, various other organic inducers such as syringaldazine, thiamine $\mathrm{HCl}$, 2,6-dimethoxyphenol (DMP), and lignin were incorporated in Kirk's medium. All inducers were filter-sterilized except lignin and added into the medium.

\section{RESULTS AND DISCUSSION}

Extracellular laccase activity was observed in 16 isolated fungal species. Brown colored halos around fungal mycelium in guaiacol containing PDA medium were examined for the presence of laccase production potential by isolates (Fig. 1). NS-6 designated isolate was found to be the best among the 16 isolates with good brown

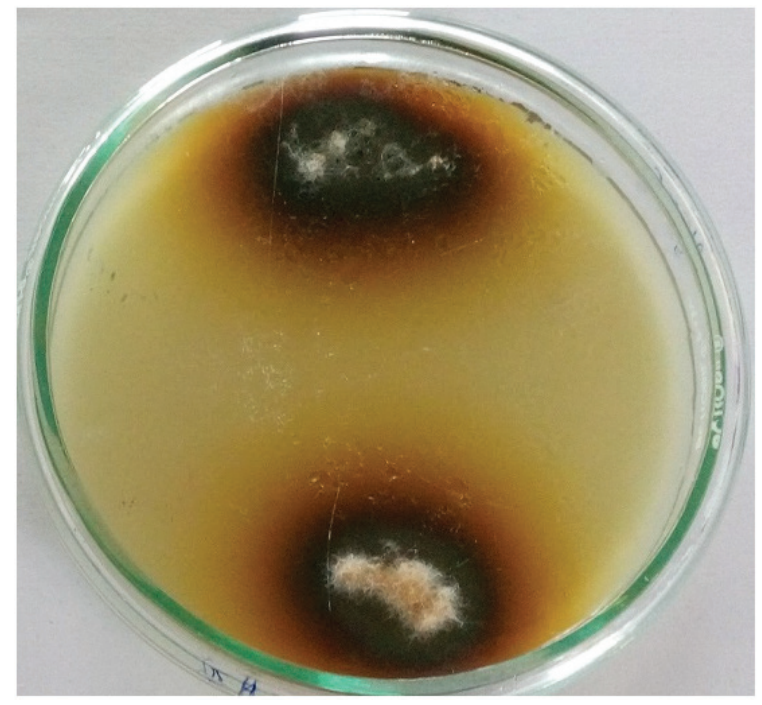

Figure 1: A. alternata with brown halos formation in guaiacol-containing plate. 
halos formation. Thus, this screening helps us to select the most promising fungal isolate. One of the observations showed reduced mycelium growth when incubated with guaiacol containing solid medium, which may be due to the changes in some metabolic activity of isolates during growth in selected media [23].

The identification of NS- 6 was further confirmed with the studies on its $18 \mathrm{~S}$ rRNA sequencing, carried out by Chromous Biotech, India. The isolate was identified as A. alternata NS-6 (Table 1). The sequence was submitted in the GenBank database (accession no. MF348243). Observation of Basic Local Alignment Search Tool (BLAST) analysis of the amplificons indicates the highest similarities with over $99 \%$ sequences generated from Alternaria strains. The phylogenetic tree was prepared by the neighborjoining (N-J) method using MEGA6 software (Fig. 2). Here, 5.8S rDNA sequence was used to construct phylogenetic tree for $A$. alternata. The result revealed that NS-6 strain was closely related to Alternaria brassicae and Alternaria brassicicola (Fig. 2).

There is a general increase in temperature observed due to the respiration of organisms. It is observed as an important factor during enzyme production and fungal growth and has a major impact during scale-up process at industrial level [24]. $A$. alternata has good laccase activity at the temperature of $30^{\circ} \mathrm{C}$ (Fig. 3). Many research articles also noticed high laccase activity for A. alternata with $35^{\circ} \mathrm{C}$ incubation temperature [25]. The
$\mathrm{pH}$ is one of the crucial parameters for fungal cultivation. The optimum $\mathrm{pH}$ for the laccase oxidation of ABTS was 4.5 with reduction of activity at high $\mathrm{pH}$. It may have an optimal $\mathrm{pH}$ range of 3.5-5.5 (Fig. 4). This may be due to the modification of catalytic site of enzyme with variation in $\mathrm{pH}$ [24]. A. alternata has also shown a high laccase activity at $\mathrm{pH} 5$ [25].

Different types of carbon and nitrogen sources in suitable amount on fungal adaptation are important for the production of laccase. Lignolytic enzyme production is also influenced by nature and concentration of nitrogen source as powerful nutritional factor by wood-rotting fungi [24]. Lignolytic enzyme production has a vital role in the growth of fungus and helps to degrade lignin present in solid lignocellulosic substrate [26]. Different sugar sources show different laccase activities. Present study showed that glucose $(1 \%)$ has an influence on laccase activity which may have an effect on growth also (Fig. 5). It also showed good laccase activity with sucrose $(1 \%)$ which may increase the chances of the use of waste industrial molasses for industrial laccase production. A variety of nitrogen sources helps to induce laccase activity with the use of its significant amount. A. alternata with $2.4-\mathrm{mM}$ ammonium sulfate has a considerable effect on laccase activity. Ammonium nitrate also has a remarkable impact on laccase activity (Fig. 6). Decolorization of effluent was also affected by a variety of nitrogen sources by researchers [27].

Table 1: Identification of fungal isolate.

\begin{tabular}{|c|c|c|}
\hline Organism & Accession No. & Sequence \\
\hline A. alternata & MF348243 & $\begin{array}{l}\text { TGAGGTAACCTCTCGGGGTTACAGCCTTGCTGAATTATTCACCCTTGTCTTTTGCGTACTTCTTGTTTCCTTGGTGGGTTCGCCCACCACT } \\
\text { AGGACAAACATATAAACCTTTTGTAATTGCAATCAGCGTCAGTAACAAATTAATAATTACAACTTTCAACAACGGATCTCTTGGTTCTGGC } \\
\text { ATCGATGAAGAACGCAGCGAAATGCGATAAGTAGTGTGAATTGCAGAATCAGTGAATCATCGAATCTTTGACGCACATTGCGCCCTTT } \\
\text { GGTATTCCAAAGGGCATGCCTGTTCGAGCGTCATTTGTACCCTCAAGCTTTGCTTGGTGTTGGGCGTCTTGTCTCTAGCTTGCTGGAGAC } \\
\text { TCGCCTTAAAGTAATTGGCAGCCGGCCTACTGGTTTCGGAGCGCAGCACAAGTCGCACTCTCTATCAGCAAAGGTCTAGCATCCATTAAG } \\
\text { CCTTTTTTTCAACTTTTGACCTCGGATCAGGTAGGGATACCCGCTGAACTTAAGCATATCAATAAGCGGAGGAAA }\end{array}$ \\
\hline
\end{tabular}

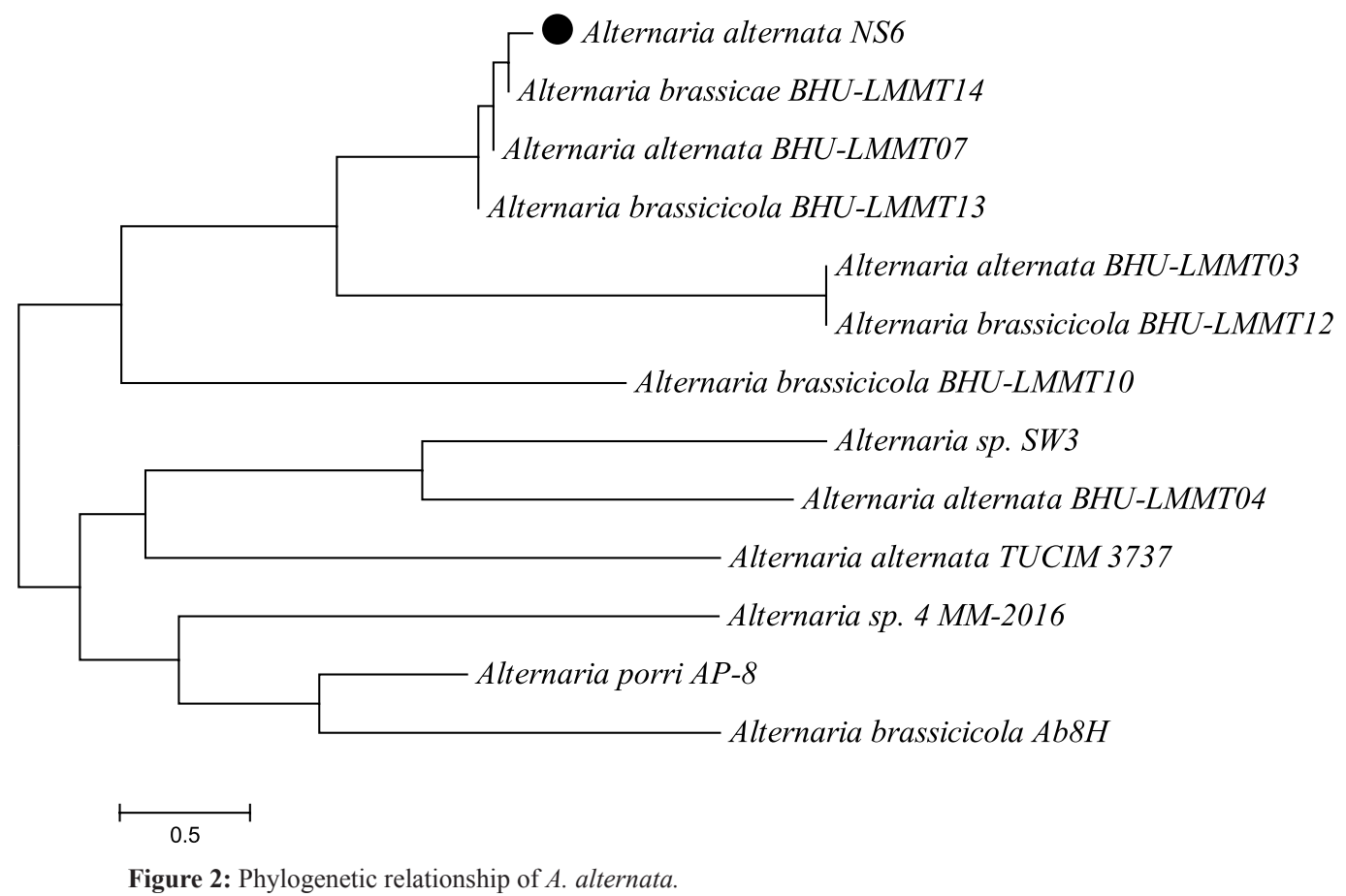




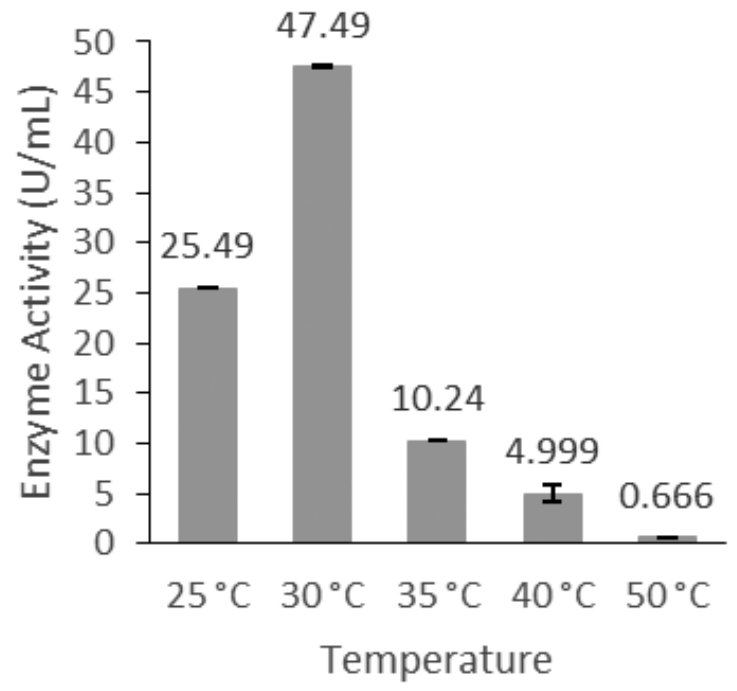

Figure 3: Effect of temperature on laccase activity.

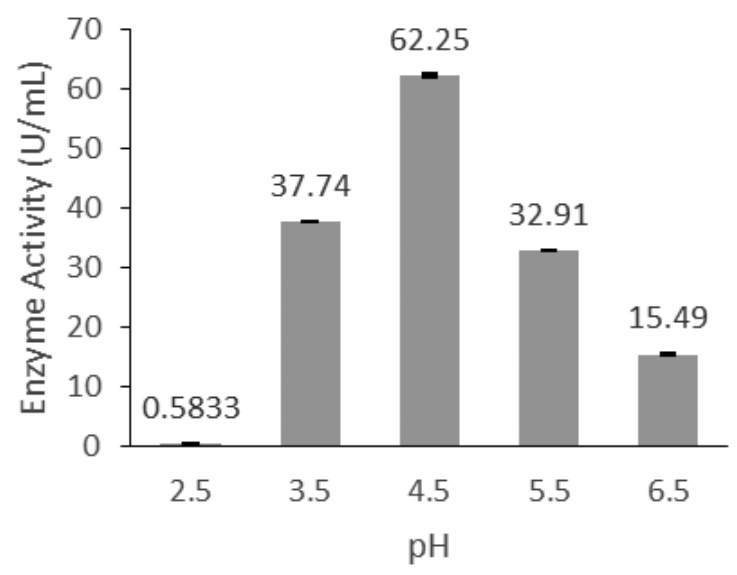

Figure 4: Effect of $\mathrm{pH}$ on laccase activity.

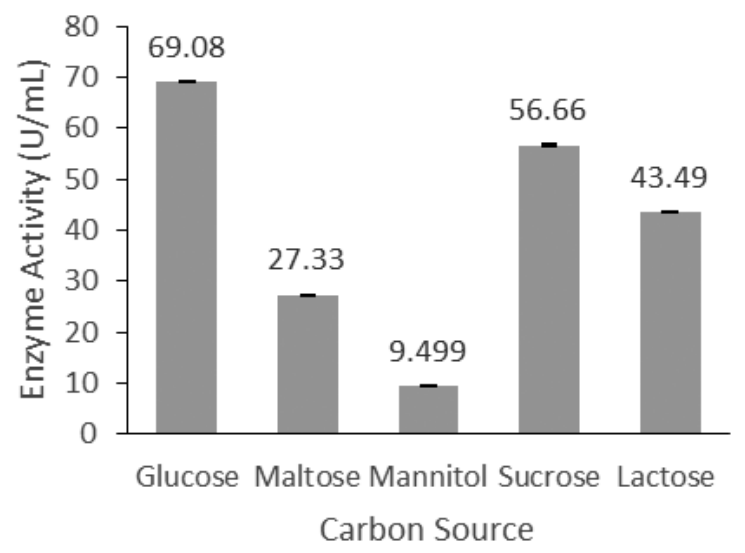

Figure 5: Effect of carbon source on laccase activity.

Aromatic compounds help to induce the production of laccase. Veratryl alcohol had an inductive effect during optimization process with $273.32 \mathrm{U} / \mathrm{ml}$ laccase activity. DMP also showed

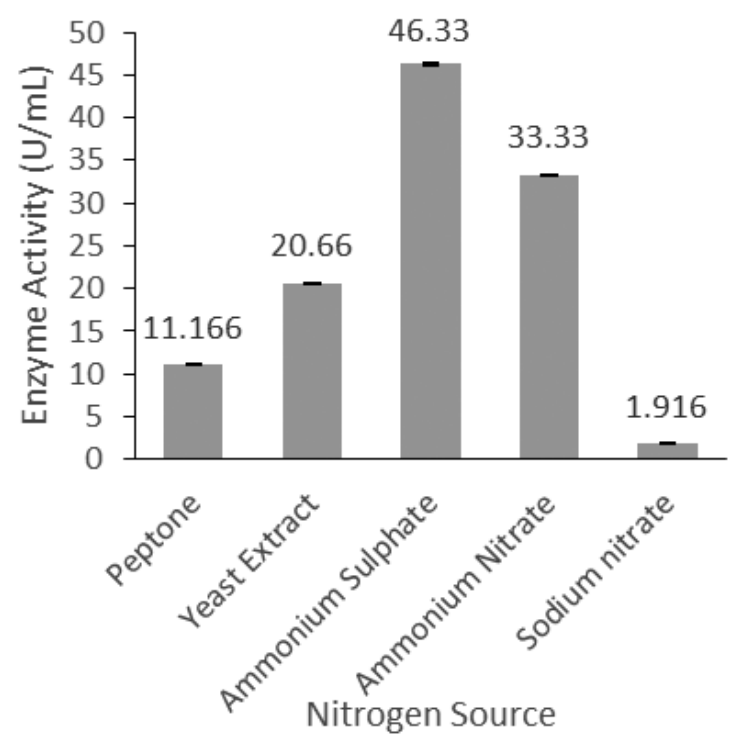

Figure 6: Effect of nitrogen sources on laccase activity.

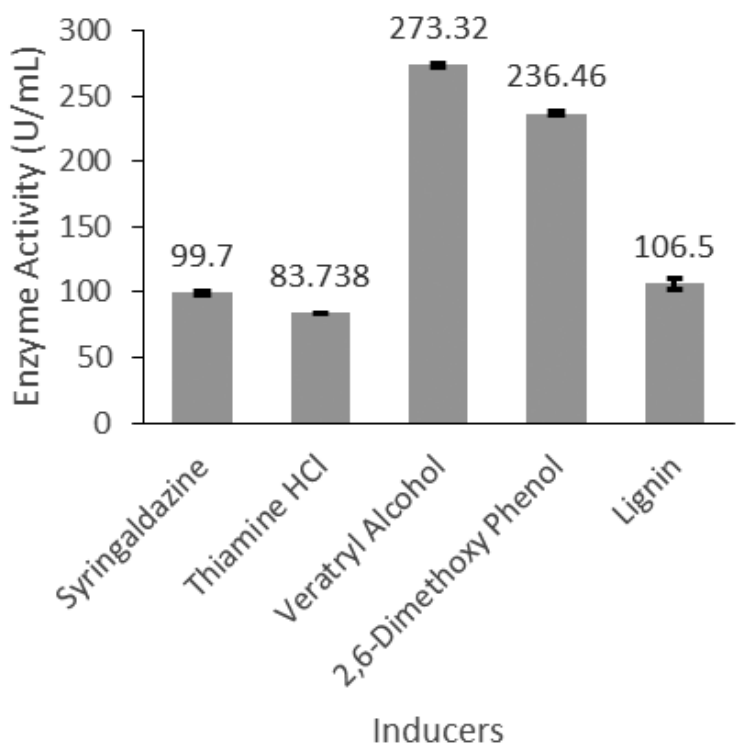

Figure 7: Effect of inducers on laccase activity.

a positive effect on laccase production (Fig. 7). Arora and Gill (2001) have studied the influence of veratryl alcohol on laccase production in Ganoderma lucidum, Trametes versicolor, and Dichomitus squalens [28]. The highest laccase activity was $72.13 \mathrm{U} / \mathrm{ml}$, observed with $\mathrm{Cu}^{+2}$ after 9 days of incubation (Fig. 8). Regulation of laccase expression can be observed with the use of selected metal ions. Affinity for different metal ions was recorded for different species. Laccase activity in almost all fungi can be influenced by copper in $T$. versicolor, Ceriporiopsis subvermispora, Pleurotus ostreatus, and Trametes pubescens. At the transcription level, copper-mediated regulation was observed [29]. $\mathrm{Fe}^{+2}$ is also reported as an important cation to induce laccase activity in Pleurotus eryngii [30] and Trametes velutina [31]. 


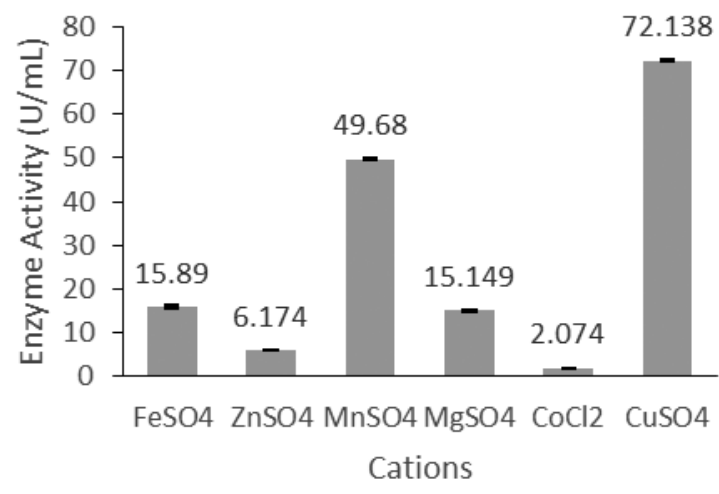

Figure 8: Effect of cations on laccase activity.

\section{CONCLUSION}

The present study illustrates that $A$. alternata NS-6 has the ability to produce brown halos on guaiacol $(0.02 \%)$ containing media. Various production parameters such as $\mathrm{pH}$, temperature, carbon, nitrogen source, cation, and organic inducers have a potential effect on laccase production. The final laccase activity obtained $273.32 \mathrm{U} / \mathrm{ml}$, with a supplementation of $1.5 \mathrm{mM}$ veratryl alcohol.

\section{CONFLICT OF INTEREST}

The authors declare that they do not have any conflicts of interest.

\section{FINANCIAL SUPPORT}

None.

\section{REFERENCES}

1. Amutha C, Abhijit M. Screening and isolation of laccase producers, determination of optimal condition for growth, laccase production and choose the best strain. J Bioremed Biodegredation 2015; 6(4):1-8; doi:10.4172/2155- 6199.1000298

2. More SS, Renuka PS, Malini S. Isolation, purification, and characterization of fungal laccase from Pleurotus sp. Enzyme Res 2011; 2011:1-7. doi:10.4061/2011/248735

3. Viswanath B, Chandra MS, Pallavi H, Reddy BR. Screening and assessment of laccase producing fungi isolated from different environmental samples. Afr J Biotechnol 2008; 7(8):1-21.

4. Brijwani K, Rigdon A, Vadlani PV. Fungal laccases: production, function, and applications in food processing. Enzyme Res 2010; 2010:1-10; doi:10.4061/2010/149748

5. Alfarra HY, Hasali NHM, Omar MN. A lignolytic fungi with laccase activity isolated from Malaysian local environment for phytochemical transformation purposes. Int Res J Biol Sci 2013; 2(2):51-4.

6. Shrestha P, Joshi B, Joshi J, Malla R, Sreerama L. Isolation and physicochemical characterization of laccase from Ganoderma lucidum-CDBT1 isolated from its native habitat in Nepal. BioMed Res Int 2016; 2016:1-10; doi:10.1155/2016/3238909

7. D'Souza TM, Boominathan K, Reddy CA. Isolation of laccase gene-specific sequences from white rot and brown rot fungi by PCR. Appl Environ Microbiol 1996; 62(10):3739-44; doi:00992240/96/\$04.0010

8. Vantamuri AB, Adhoni SA, Nadaf PD, Payamalle S, Guruvin SK, Manawadi SI. Isolation and characterization of laccase producing fungi from different environmental samples. Int J Recent Sci Res 2015; 6:6853-7.

9. White TJ, Bruns T, Lee SJWT, Taylor J. Amplification and direct sequencing of fungal ribosomal RNA genes for phylogenetics. PCR Protocol: Guide Methods Appl 1990; 18(1):315-22.
10. Gardes M, Bruns TD. ITS primers with enhanced specificity for basidiomycetes-application to the identification of mycorrhizae and rusts. Mol Ecol 1993; 2(2):113-8.

11. Bruno WJ, Socci ND, Halpern, AL. Weighted neighbor joining: a likelihood-based approach to distance-based phylogeny reconstruction. Mol Biol Evol 2000; 17(1):189-97.

12. Paice MG, Reid ID, Bourbonnais R, Archibald FS, Jurasek L. Manganese peroxidase, produced by Trametes versicolor during pulp bleaching, demethylates and delignifies kraft pulp. Appl Environ Microbiol 1993; 59(1):260-5; doi:0099-2240/93/010260-06\$02.00/0

13. Thakur S, Shrivastava B, Ingale S, Kuhad RC, Gupte A. Degradation and selective ligninolysis of wheat straw and banana stem for an efficient bioethanol production using fungal and chemical pretreatment. 3 Biotech 2013; 3(5):365-72; doi:10.1007/s13205-0120102-4

14. Vares T, Kalsi M, Hatakka A. Lignin peroxidases, manganese peroxidases, and other ligninolytic enzymes produced by Phlebiaradiata during solid-state fermentation of wheat straw. Appl Environ Microbiol 1995; 61(10):3515-20; doi:0099-2240/95/\$04.0010

15. Kandasamy S, Muniraj IK, Purushothaman N, Sekar A, Sharmila DJS, Kumarasamy R, Uthandi S. High level secretion of laccase $(\mathrm{LccH})$ from a newly isolated white-rot basidiomycete, Hexagoniahirta MSF2. Front Microbiol 2016; 7:1-11; doi:10.3389/fmicb.2016.00707

16. Risdianto H, Sofianti E, Suhardi SH, Setiadi T. Optimization of laccase production using white rot fungi and agricultural wastes in solid-state fermentation. J Eng Technol Sci 2012; 44(2):93-105; doi:10.5614/itbj. eng.sci.2012.44.2.1

17. Jaber SM, Shah UKM, Asa' ari AZM, AriffAB. Optimization of laccase production by locally isolated Trichoderma muroiana IS1037 using rubber wood dust as substrate. BioResources. 2017; 12(2):3834-49.

18. Atalla M, Zeinab H, Eman R, Amani A, Abeer A. Screening of some marine-derived fungal isolates for lignin degrading enzymes (LDEs) production. Agricul Biol J North Am 2010; 1(4):591-9.

19. Järvinen J, Taskila S, Isomäki R, Ojamo H. Screening of white-rot fungi manganese peroxidases: a comparison between the specific activities of the enzyme from different native producers. AMB Express 2012; 2(62):1-9; doi:10.1186/2191-0855-2-62

20. Kalmis E, Yasa I, Kalyoncu F, Pazarbasi B, Koçyigit A. Ligninolytic enzyme activities in mycelium of some wild and commercial mushrooms. Afr J Biotechnol 2008; 7(23):4314-20.

21. El-Aty AAA, Mostafa FA. Effect of various media and supplements on laccase activity and its application in dyes decolorization. Malaysian J Microbiol 2013; 9(2):166-75; doi:10.21161/mjm.47712

22. Desai SA. Isolation and characterization of Laccase producing bacteria from contaminated sites. Biosci Discov 2017; 8:567-73.

23. Kaur H, Kapoor S, Sharma S. An efficient method for qualitative screening of ligninolytic enzyme potential of Ganoderma lucidum. Int J Curr Microbiol Appl Sci 2018; 7(8):2442-59; doi:10.20546/ ijcmas.2018.708.247

24. Patel H, Gupte A, Gupte S. Effect of different culture conditions and inducers on production of laccase by a basidiomycete fungal isolate Pleurotus ostreatus HP-1 under solid state fermentation. BioResources 2009; 4(1):268-84.

25. Irfan M, Mehmood S, Irshad M, Anwar Z. Optimized production, purification and molecular characterization of fungal laccase through Alternaria alternata. Turkish J Biochem 2018; 43(6):613-22; doi:10.1515/tjb-2017-0239

26. Onyancha W. Degradation of agroresiduces with value added products by solid state fermentation with calocybe indicay. J Biol, Agricul Healthcare 2016; 6(20):27-41.

27. D'Souza-Ticlo D, Verma AK, Mathew M, Raghukumar C. Effect of nutrient nitrogen on laccase production, its isozyme pattern and effluent decolorization by the fungus NIOCC\# 2a, isolated from mangrove wood. Indian J Marine Sci 2006; 35(4):364-72.

28. Arora DS, Gill PK. Effects of various media and supplements on laccase production by some white rot fungi. Bioresour Technol 2001; 77(1):89-91; doi:10.1016/S0960-8524(00)00114-0 
29. Zhu C, Bao G, Huang S. Optimization of laccase production in the white-rot fungus Pleurotus ostreatus (ACCC 52857) induced through yeast extract and copper. Biotechnol Biotechnological Equip 2016; 30(2):270-6; doi:10.1080/13102818.2015.1135081

30. Stajić M, Vukojević J. Interaction of trace elements and ligninolytic enzymes in Pleurotus eryngii. Biol Trace Elem Res 2011; 143(2):12028; doi:10.1007/s12011-010-8918-4

31. Yang Y, Wei F, Zhuo R, Fan F, Liu H, Zhang C, et al. Enhancing the laccase production and laccase gene expression in the white-rot fungus Trametes velutina 5930 with great potential for biotechnological applications by different metal ions and aromatic compounds. PLoS One 2013; 8(11):e79307; doi:10.1371/journal.pone.0079307
How to cite this article:

Thakkar AT, Bhatt SA. Isolation, identification and optimization of laccase from Alternaria alternata. J Appl Biol Biotech 2020;8(03):064-069. DOI: 10.7324/ JABB. 2020.803012 